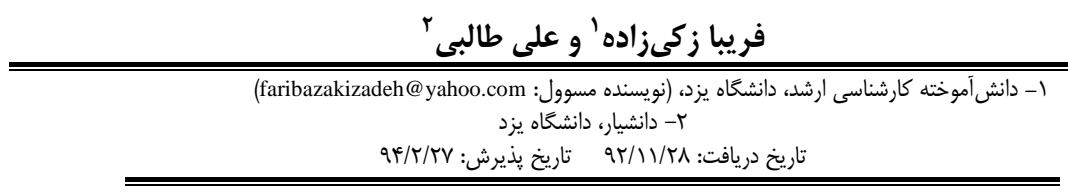

קكيده

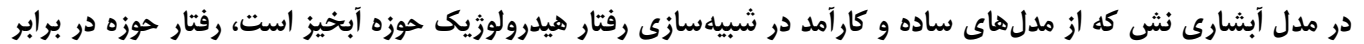

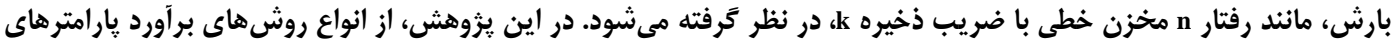

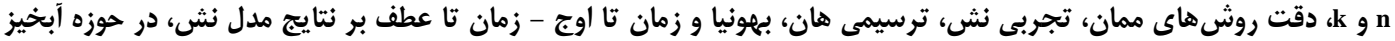

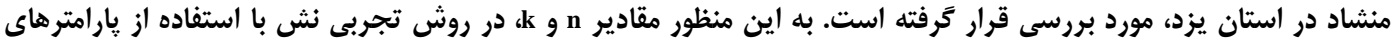

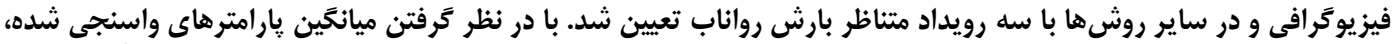

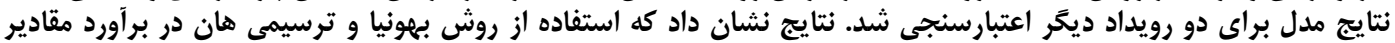

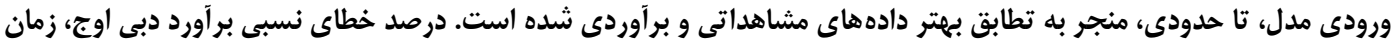

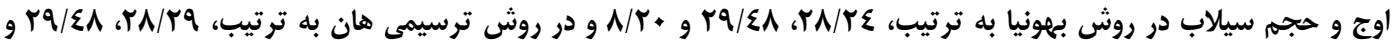

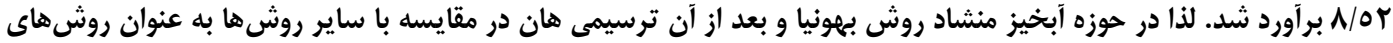

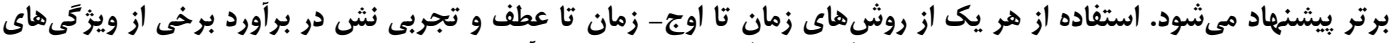

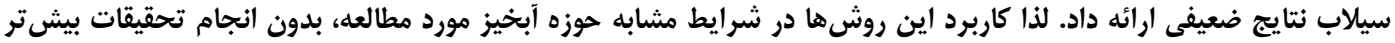

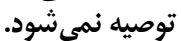

وازههاى كليدى: مدلسازى بارش- رواناب، روش بهونيا، مدل نش، حوزه آبخيز منشاد، هيدروَّراف واحد لحظهاى

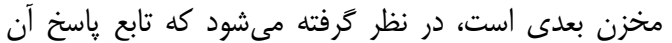

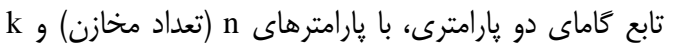

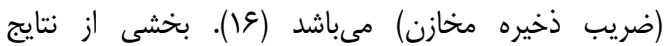

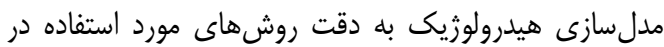

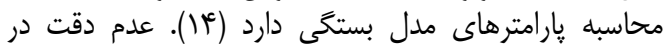

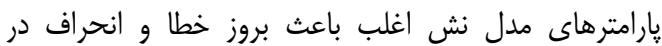

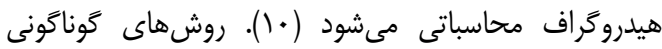

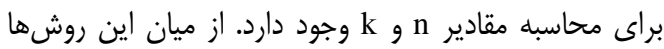

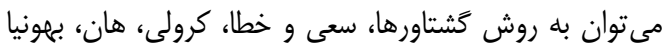

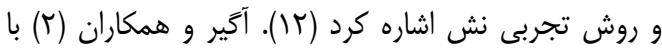

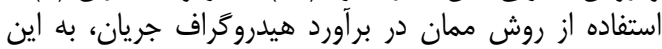

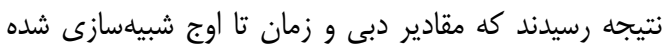

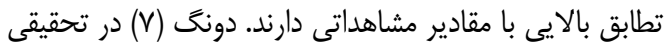

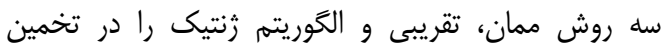

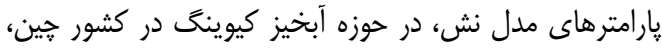

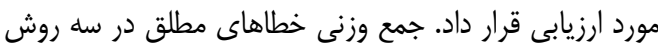

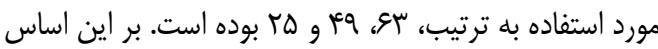

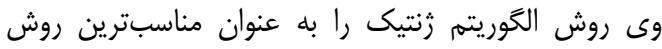

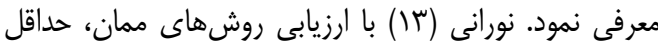

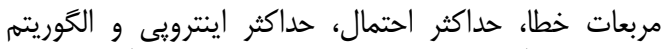

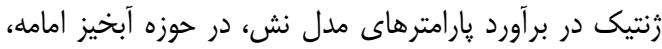

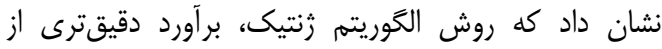

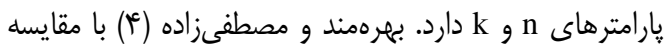

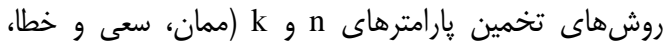

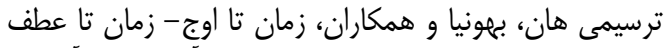
و تجربى نش) بر نتايج مدل نش در حوزه آبخيز جعفرآباد، بهان 1- Nash's Linear Reservoir Model
فرايند بارش- رواناب بديدهاى يِيجيده است كه عوامل

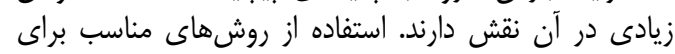

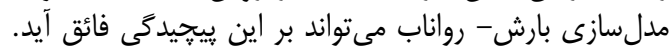

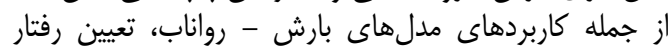

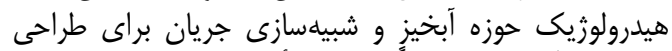

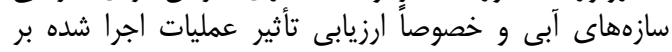

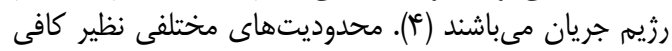

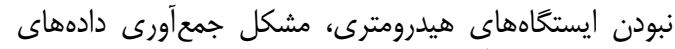

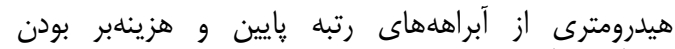

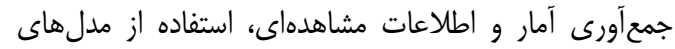

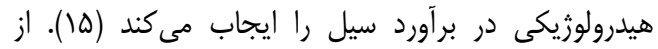

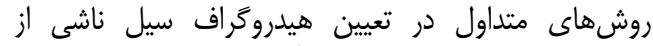

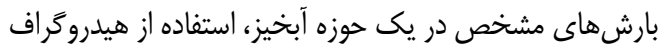

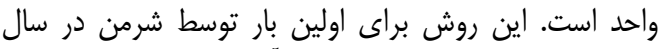

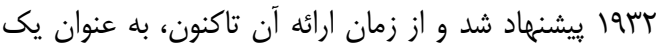

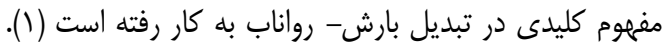

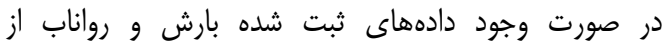

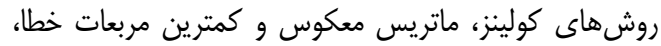

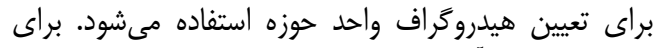

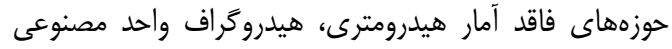

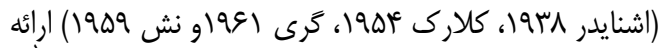

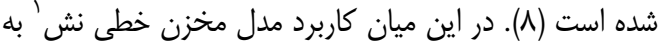

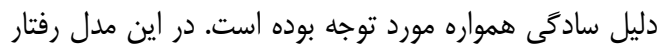

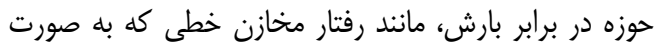

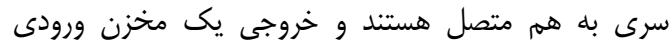


حوزه آبخيز منشاد از نظر تقسيمات كشورى جز استان يزد إندا

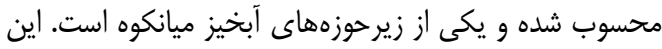

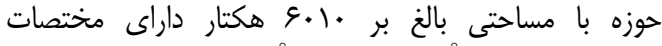

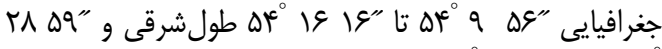

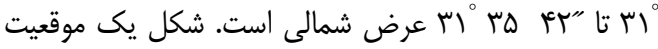

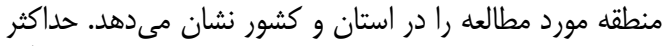

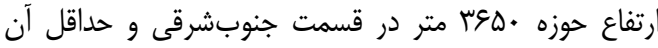

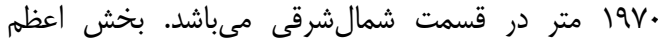

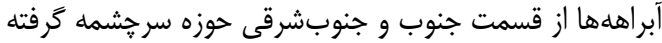

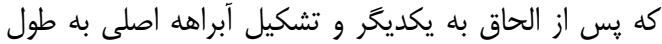

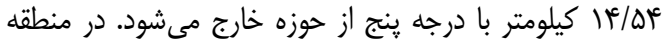

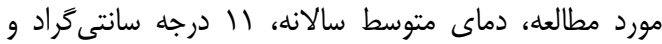

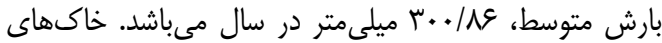

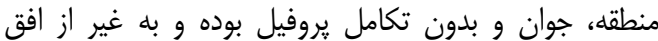

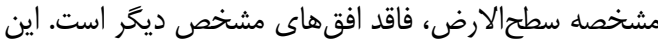

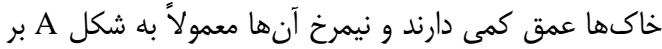

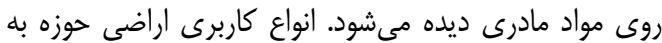

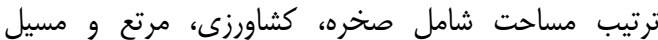
مىباشد. شيب متوسط منطقه مورد مطالعه
اين نتيجه رسيدند كه در روشهاى ممان و سعى و خطا،

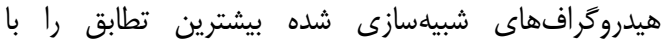

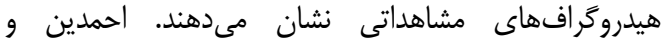

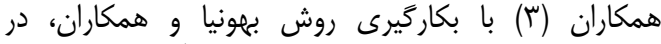

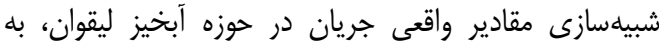

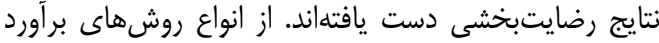

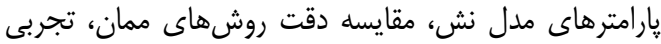

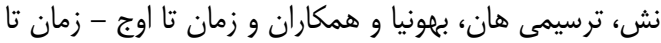

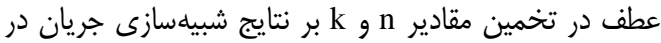

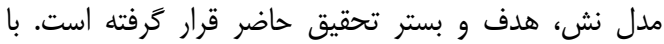

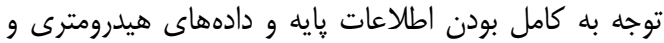

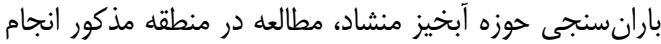

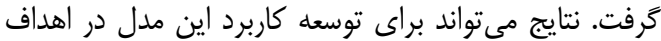

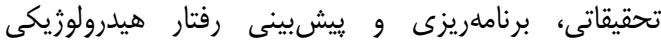
حوزههاى آبخيز به منظور مديريت سيلاب مورد استفاده قرار كيرد.

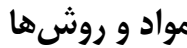 معرفى منطقه مورد مطالعه - مواد}

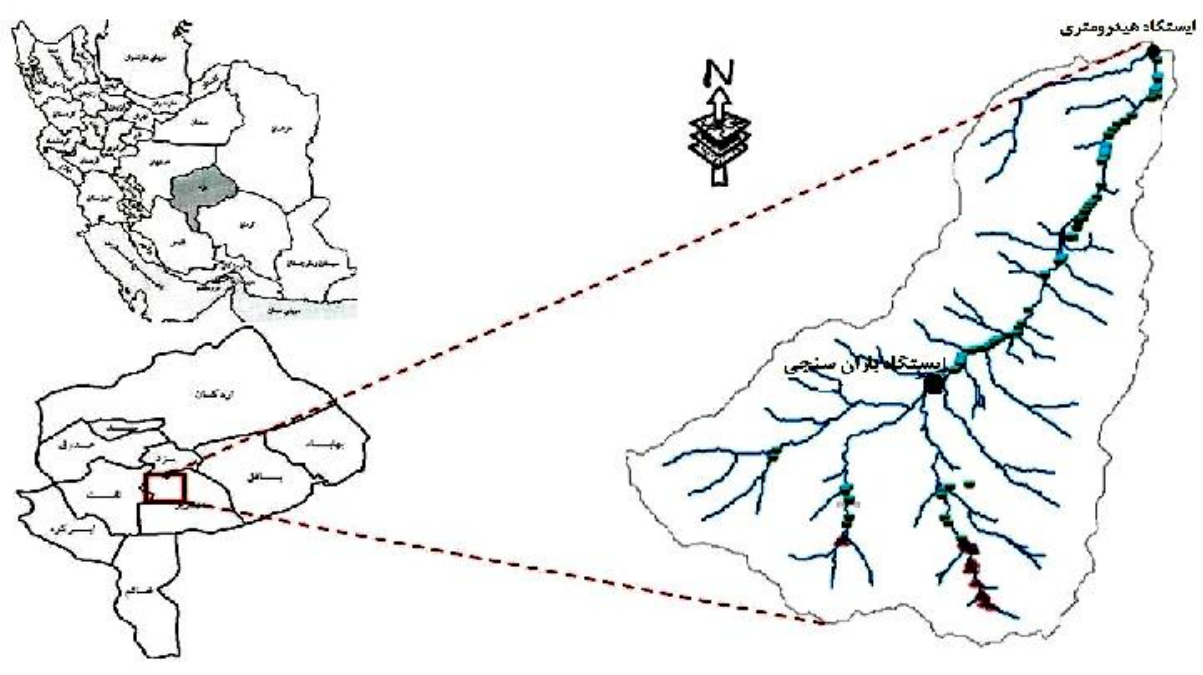

شكل 1- موقعيت منطقه مورد مطالعه در استان يزد و ايران

Figure 1. The location of study area in Yazd province and Iran

است. يُ از جمعآورى آمار و اطالاعات بارش- رواناب،

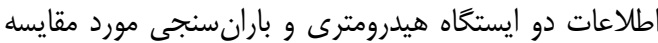

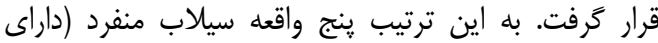

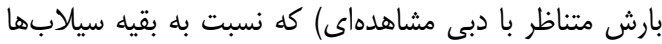

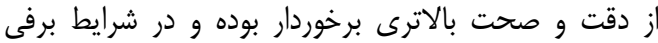

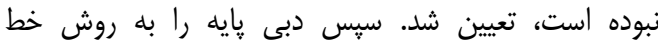

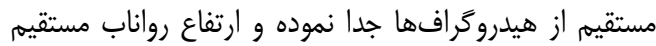
براى هر سيلاب مشخص شد. در نهايت، هايتوكراف اف بارش
روش تحقيق مراحل كلى انجام اين يزوهش، به به طور خلاصه در شكل

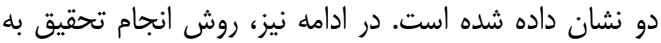

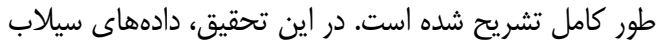

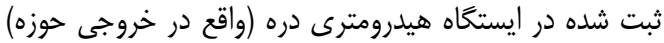

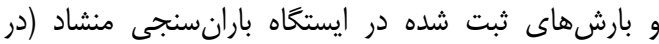

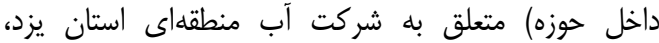
جمع آورى شده و در تجزيه و تحليل مورد استفاده قرار كرفتهن 


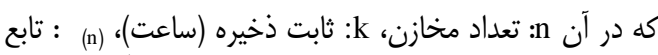
كاماى n، t زمان (ساعت) و

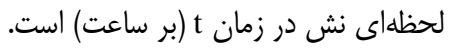

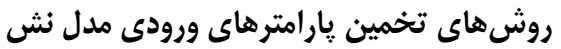

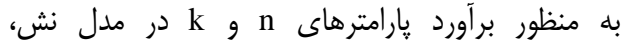

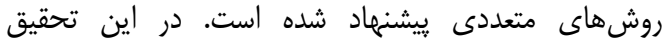
روش هاى زير مورد استفاده قرار گرفته است: روش ممانها

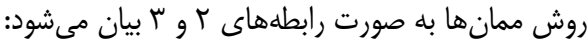

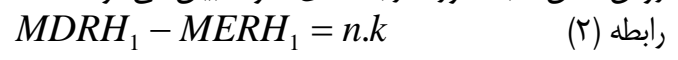

رابطه (r)

$M D R H_{2}-M_{E R H_{2}}=n(n+1) k^{2}+2 n k\left(M E R H_{1}\right)$

كه در آن

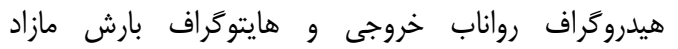

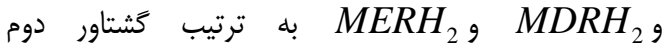
هيدروگراف رواناب خروجى و هايتوگراف بارش مازاد هستند

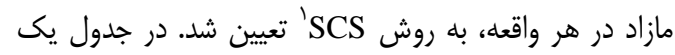

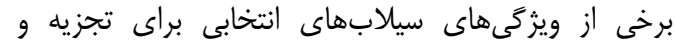
تحليل، ارائه شده است.

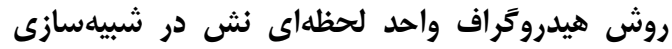
جريان سطحى در حوزه آبخيز

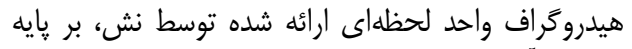

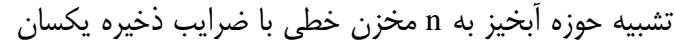

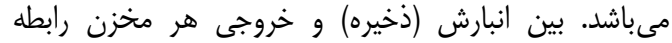

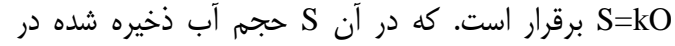

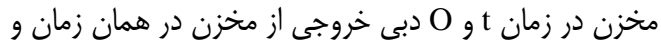

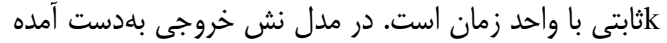

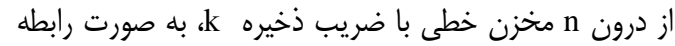

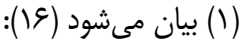

$O_{n}=\frac{1}{k \Gamma_{(n)}}\left(\frac{t}{k}\right)^{n-1} e^{-\frac{t}{k}}$

Table 1. Main characteristics of rainfall cases selected for analysis

جدول ا- ويزگى هاى سيلابهاى انتخابى براى تجزيه و تحليل

\begin{tabular}{|c|c|c|c|c|}
\hline مدت تداوم بارش موثر & مقدار بارش موثر & مقدار بارش اوليه & تاريخ سيلاب & شماره رويداد \\
\hline$\Delta / \Delta$ & $r / \Delta$ & $\mid r F / \Lambda$ & ITNY/Q/IQ & 1 \\
\hline $11 / \Delta$ & $N / \Delta V$ & $V Q / S$ & $1 r \Lambda T / 1 \cdot / M-19$ & r \\
\hline $1 / v a$ & .18 & $11 / \Delta$ & $1 \Gamma \wedge \Delta / 1 T / \pi F$ & r \\
\hline $9 / \% \Delta$ & $1 \cdot / 1$ & $F / T$ & 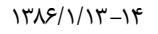 & f \\
\hline 1 &.$/ \mu$ & $\Delta / N \pi$ & $I T M / I T / V$ & a \\
\hline
\end{tabular}

روش زمان تا اوج- زمان تا عطف

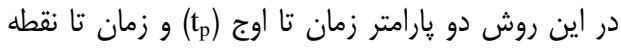

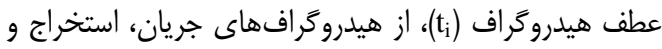

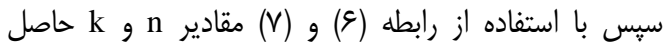
$n=\left(\frac{t_{p}}{t_{i}-t_{p}}\right)^{2}+1$ مىشود (IT). $k=\frac{t_{i}}{n-1+(n-1)^{0.5}}$
رابطه (צ)

(V) رابطه

\section{روش تجربى نش} نش بر اساس مطالعات خود در برخى از حوزههاى آبخيز

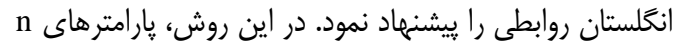

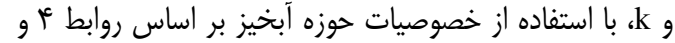
$\mathrm{n}=2.29 \mathrm{~L}^{0.1}$

$k=\frac{1.2 A^{0.3}}{L^{0.1} S^{0.3}}$

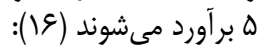

رابطه (أ) كه در آن، L: طول آبراهه اصلى (كيلومتر)، A: S: مساحت حوزه

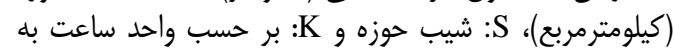
دست مى آيد. 


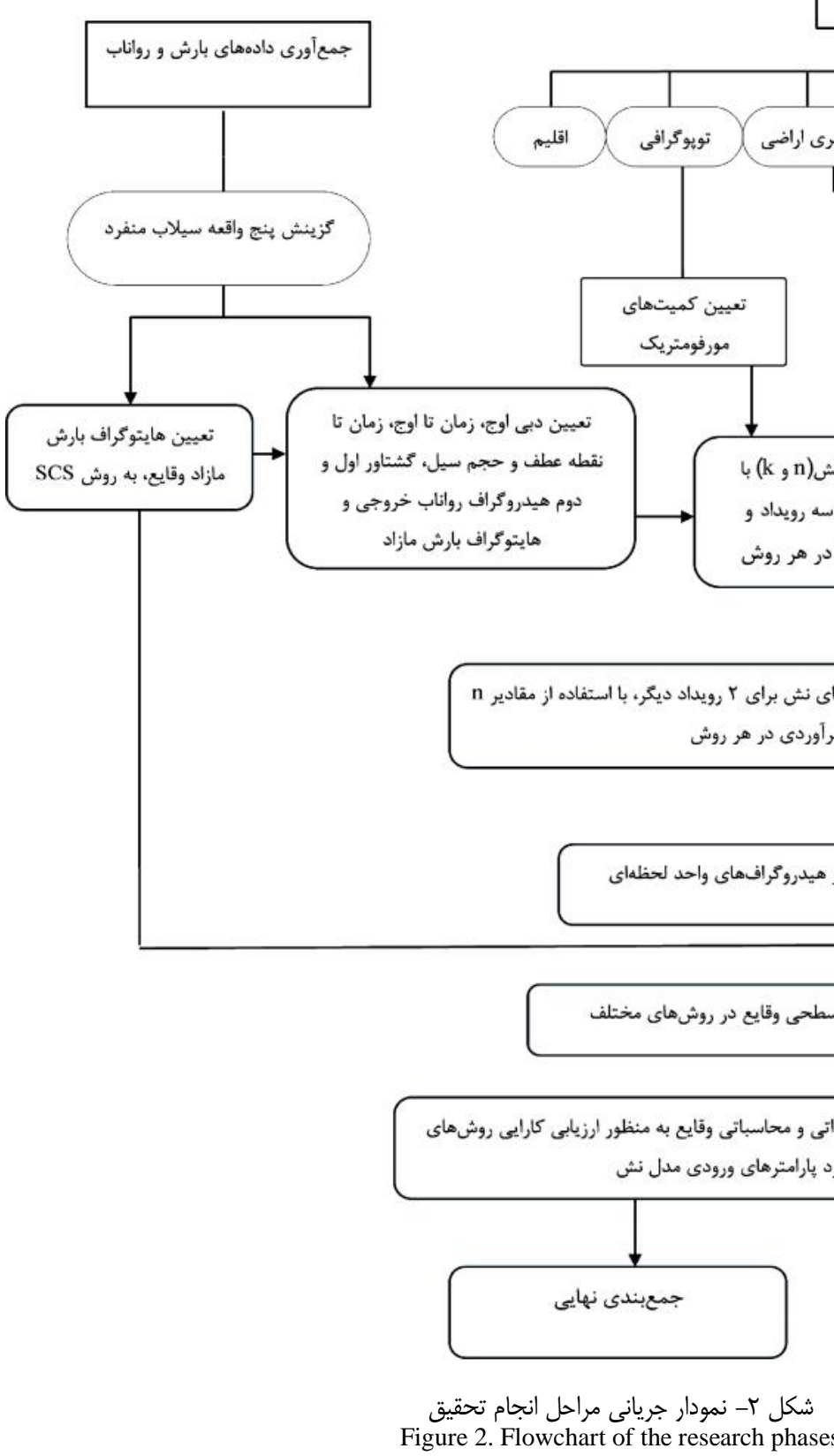

جمعآورى اطلاعات بايه 


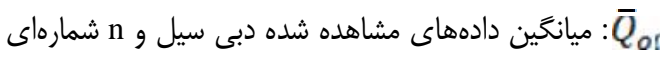

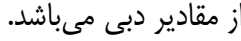

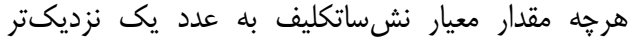

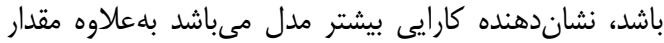

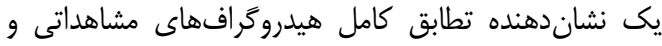

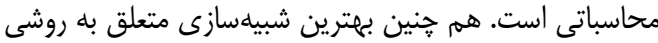

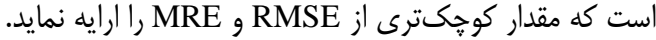

\section{نتايج و بحث}

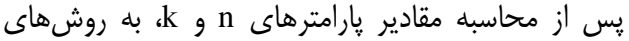

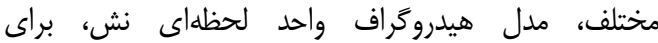

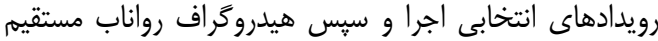

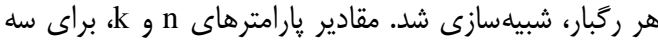

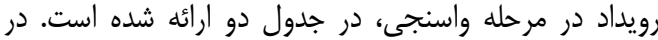

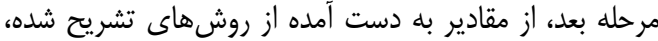

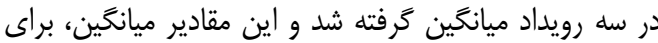

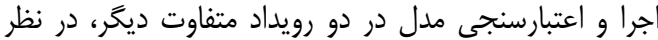

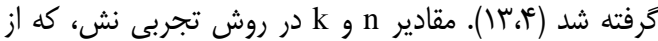

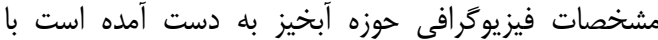

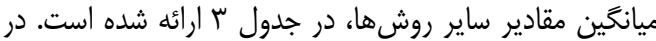

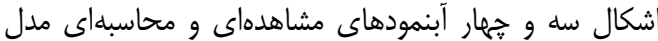

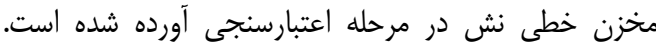

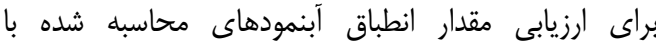

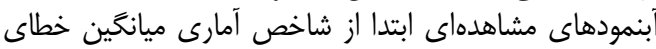

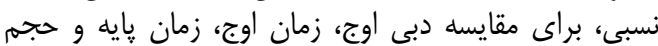

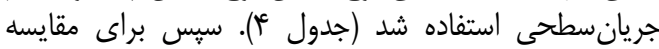

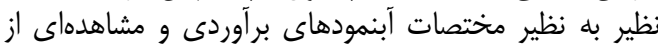
ضريب نش ساتكليف و مجذور ميانكَين مربعات خطا استفا استفاده شده است (جدول هان ).

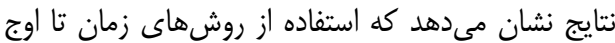

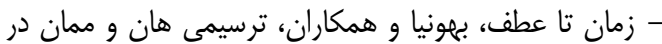

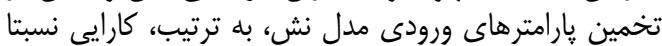

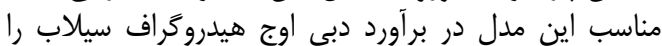

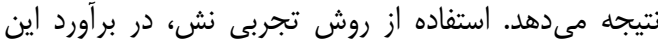

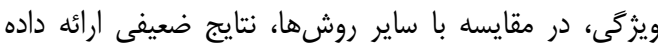

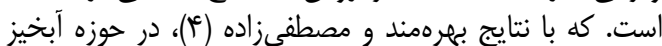

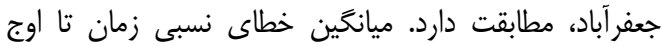

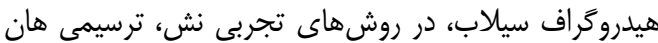

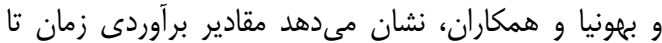

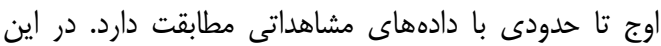

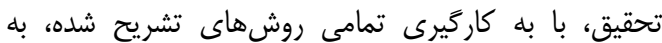

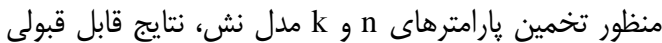

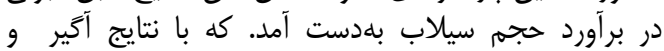

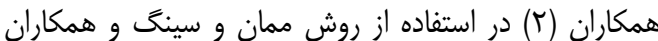

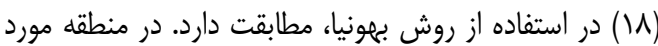

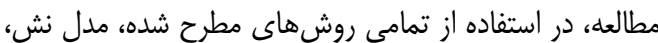

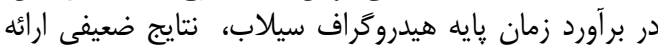

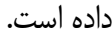

روش بهونيا و همكاران

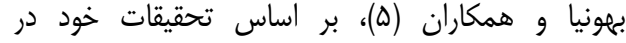

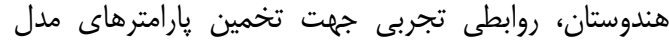
نش، به صورت زير، ييشنهاد دادندا: $n=5.53 \beta^{1.75}+1.040 .01<\beta<0.35 \quad$ (II) $n=6.29 \beta^{1.998}+1.157 \quad \beta \geq 0.35 \quad$ رابطه (I) $k=\frac{t_{p}}{n-1}$ رابطه (T)

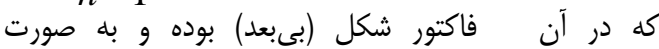

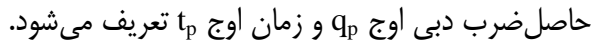

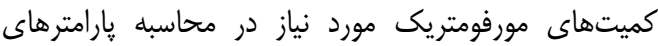

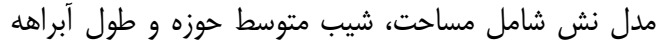

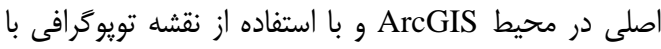

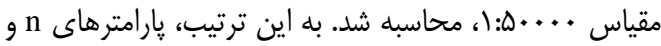

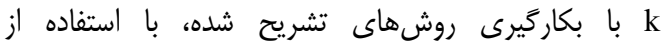

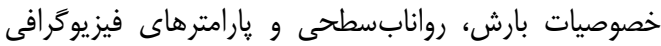
(بسته به نوع روش)، در رويدادهاى يكى تا سهان، در حوزه آبخيز

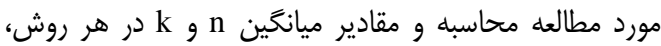

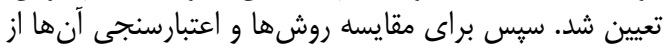

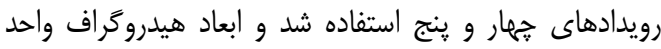

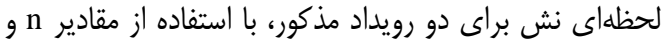

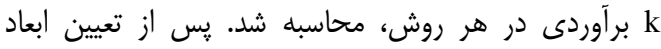

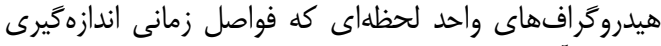

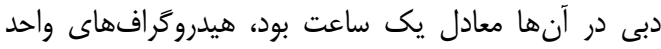

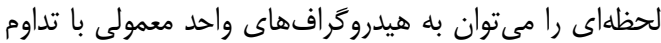

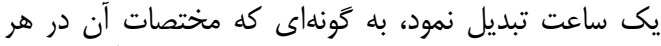

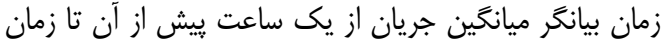

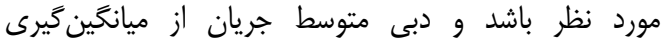

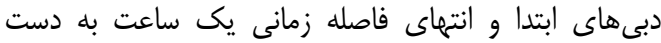

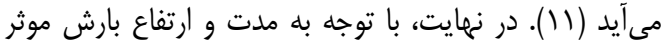

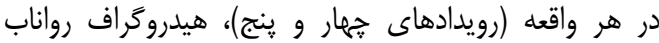

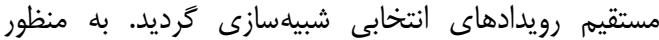

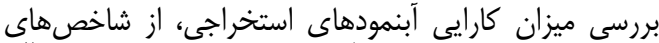

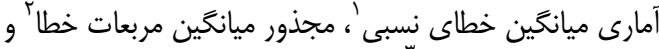

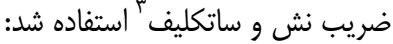

$\mathrm{RE}=\left|\frac{\mathrm{X}_{0}-\mathrm{X}_{\mathrm{e}}}{\mathrm{X}_{0}}\right| \times 100$

$\mathrm{MRE}=\frac{1}{\mathrm{n}} \sum_{\mathrm{i}=1}^{\mathrm{n}} \mathrm{RE}_{\mathrm{i}}$

RMSE $=\sqrt{\frac{\sum_{\mathrm{i}=\left(\mathrm{X}_{\mathrm{ni}}\right.}^{\mathrm{n}}\left(\mathrm{X}_{\mathrm{ei}}\right)^{2}}{\mathrm{n}}}$

$\mathrm{NS}=1-\frac{\sum_{\mathrm{i}=2}^{\mathrm{n}}\left(\mathrm{Q}_{\mathrm{oi}}-\mathrm{Q}_{\mathrm{ei}}\right)^{2}}{\sum_{\mathrm{i}=\mathrm{a}}^{\mathrm{M}}\left(\mathrm{Q}_{\mathrm{ni}}-\bar{Q}_{\mathrm{ni}}\right)^{2}}$

كه در آن XE: مقدار مشاهده شده يارامتر، X

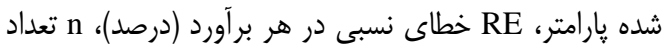

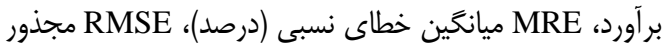

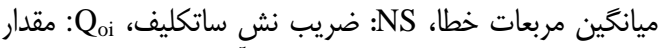

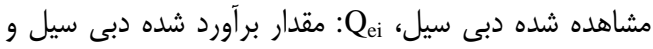


ه/ • به دست آمده است. همجنين روشهاى مورد بحث، به به به

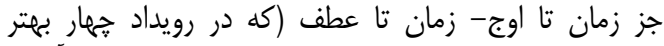

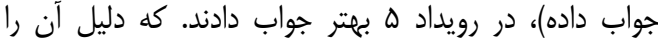

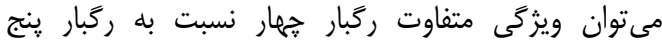

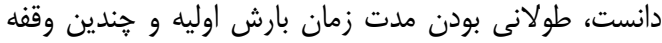

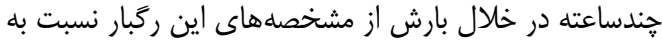

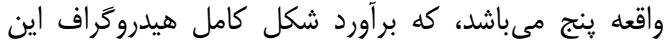

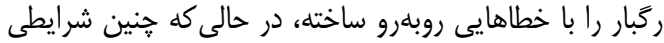
در ارتباط با رخبار ه مطرح نيست.
مقايسه نظير به نظير مختصات آبنمودهاى محاسبهاى

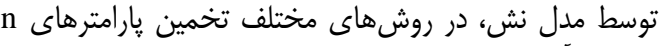

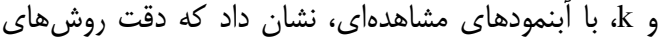

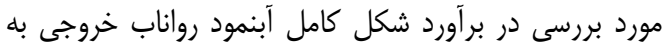

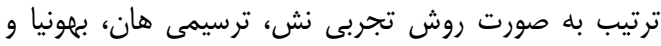

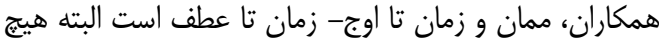

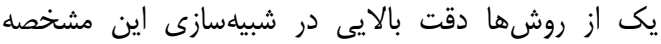

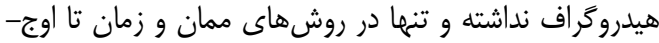

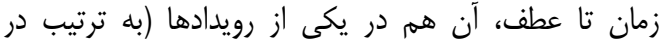
رويدادهاى جهار و پينج)، مقدار معيار نش ساتكليف ان بالاتر از

Table 2. N and K values calculated from different methods in the calibration phase

\begin{tabular}{|c|c|c|c|c|c|c|c|c|}
\hline \multicolumn{2}{|c|}{ 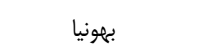 } & \multicolumn{2}{|c|}{ ترسيمى هان } & \multicolumn{2}{|c|}{ زمان تا اوج - زمان تا عطف } & \multicolumn{2}{|c|}{ 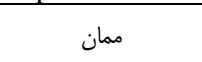 } & \\
\hline $\mathrm{k}$ & $\mathrm{n}$ & $\mathrm{k}$ & $\mathrm{n}$ & $\mathrm{k}$ & $\mathrm{n}$ & $\mathrm{k}$ & $\mathrm{n}$ & - \\
\hline.$/ r \Lambda$ & $I r / \Delta r$ & . & $15 / q 4$ & $\cdot / \Delta$ & N/II & - /AF & $0 / 19$ & 1 \\
\hline $1 / 91$ & $9 / \%$. & $1 / 90$ & $N / \Delta$ & $1 / 9 \pi$ & $1 f / v g$ & $r / r$ & $r / \Delta T$ & r \\
\hline $1 / \& \Delta$ & ש & $T / \Delta r$ & $1 \cdot / \wedge \Delta$ &.$/ 91$ & TE & $r / 1$. & $\Delta / \cdot T$ & r \\
\hline
\end{tabular}

Table 3. Average values of $\mathrm{n}$ and $\mathrm{k}$ parameters calculated from different methods

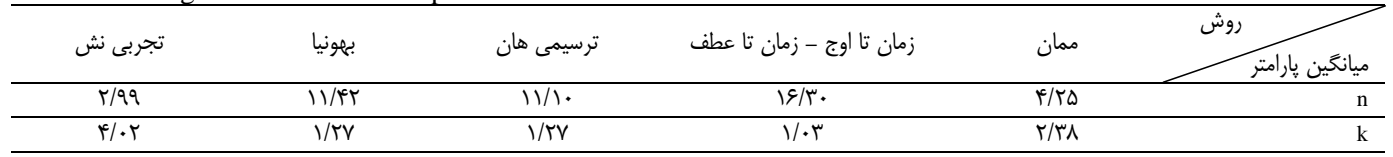

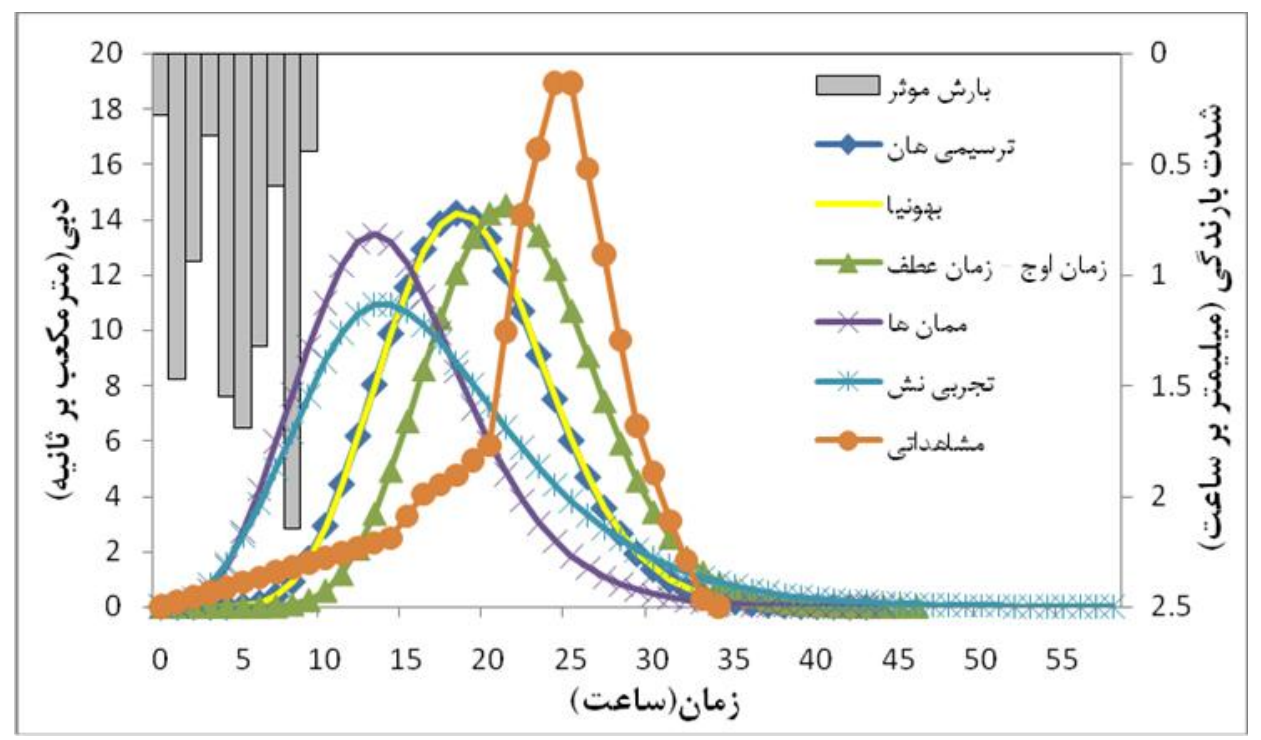

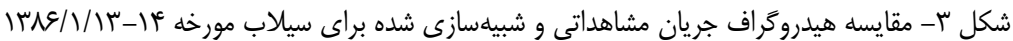

Figure 3. Comparison of observed and simulated hydrographs of different methods for the storm event of 2 April 2007 


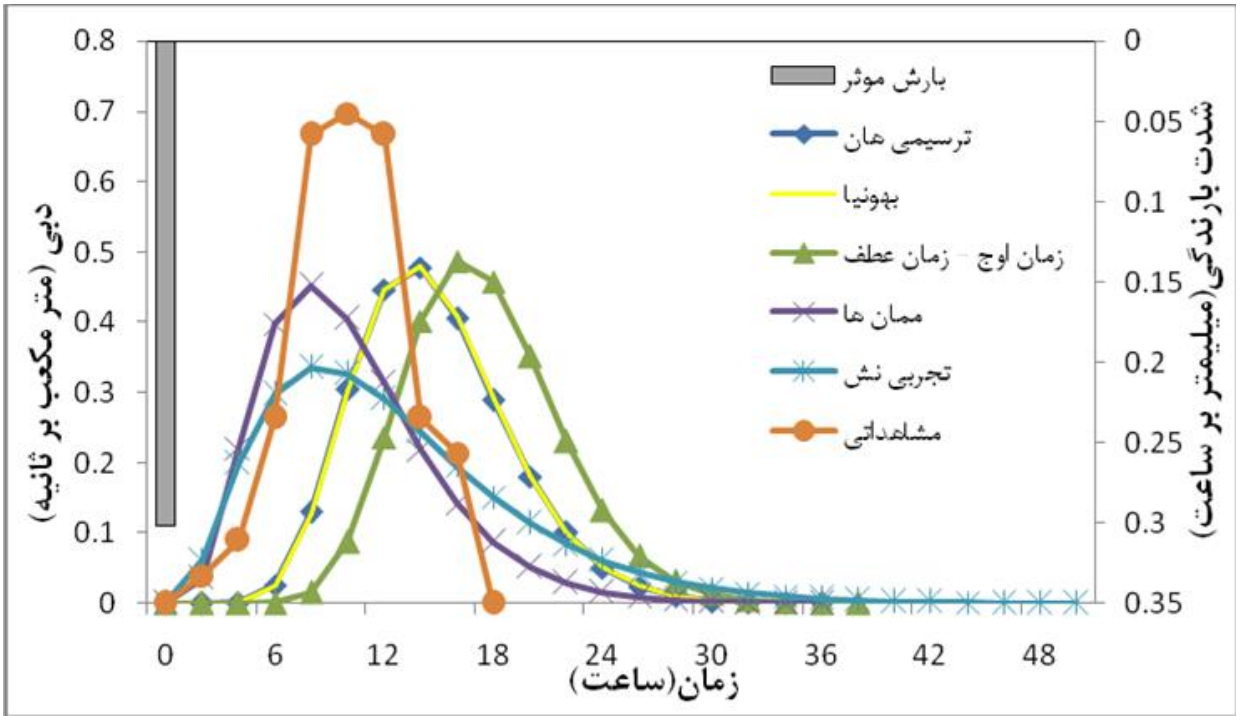

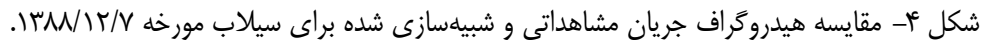

Figure 4. Comparison of observed and simulated hydrographs of different methods for the storm event of 26 February 2010

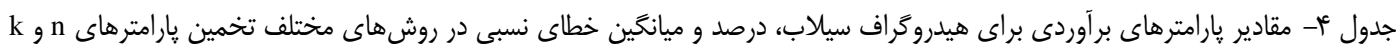
در مرحله اعتبارسنجى

Table 4. Estimated parameters values for flood hydrographs, percentage and Mean Relative Error for different methods of estimating $\mathrm{n}$ and $\mathrm{k}$ parameters in the validation phase

\begin{tabular}{|c|c|c|c|c|c|c|c|c|c|}
\hline زمان خايه نسبي (\%) & زمان (ِايه & حجم سيلاب (\%) & حجم سيلاب & خطاى نسبي زمان & زمان اوج & خطاى نسبي دبى اوج (\%) & 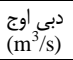 & رويداد & ل روش \\
\hline rV/qF & $\mu r / \Delta$ & $r / r F$ & $9 \mid N / 9 V$ & $r \Delta / \Lambda \mu$ & Ir & TN/9V & $1 \% / \leftarrow 9$ & $\|r N / / / M-\| F$ & \multirow{3}{*}{ ممان } \\
\hline $9 \Delta / \Lambda \mu$ & $r \Delta / r \Delta$ & $I V / \Delta A$ & IV/r & $r \cdot$ & $\wedge$ & Tr/et & $\cdot / 4 \Delta$ & IrM/IT/V & \\
\hline $81 / 19$ & - & $1 . / 99$ & - & TT/QT & - & $\Pi / V$. & - & ميانكين & \\
\hline$V \cdot / \Delta q$ & $\Delta \Lambda$ & 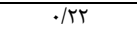 & $G Y N / / Q$ & $F \Psi / V \Delta$ & 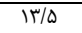 & FT/TF & $1 . / 9 \Delta$ & $\|$ W & \multirow{3}{*}{ تجربى نش } \\
\hline IVe/rq & $+q / v a$ & $1 r / v \Lambda$ & $M / \cdot r$ & 10 & $N / \Delta$ & $\Delta N / \Delta S$ & $\cdot / \mu c$ & ITM/IT/V & \\
\hline $\mid r r / F q$ & - & $\mathrm{V} / \cdot \cdot \cdot$ & - & rq/ז & - & $48 / 9$. & - & ميانكين & \\
\hline $9 / \Delta F$ & $r V / r \Delta$ & $1 / \cdot 9$ & $9 \Delta \Gamma / 91$ & $1 Y / \Delta$ & MI & אוr/ & $I F / \Delta T$ & $\|$ & \multirow{3}{*}{ زمان تا عطف } \\
\hline IOT/VA & $F \Delta / \Delta$ & $1 \% / 91$ & $10 / 19$ & 90 & $18 / 0$ & $r \cdot / 1 r$ &.$/ 19$ & $I T M / I T / V$ & \\
\hline$\Lambda / / \backslash$ & - & $V / \cdot r$ & - & $r N / V \Delta$ & - & $r \varepsilon / V \Delta$ & - & ميانكين & \\
\hline$r \Delta / V^{4}$ & $F T / V Q$ & $1 / 8 \wedge$ & STA/AD & Tr/QS & IN/TD & $r \Delta / \cdot 1$ & $|f / r|$ & IrN/M/Ir_If & \multirow{3}{*}{ ترسيمى هان } \\
\hline $91 / 8 \mathrm{~V}$ & $\mu F / \Delta$ & $1 \omega / \Gamma \Delta$ & $\mid V / 89$ & ra & $1 \% / \Delta$ & 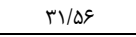 & $\cdot / 4 \wedge$ & $\mid r M / I T / V$ & \\
\hline$\Delta N / V$. & - & $N / \Delta T$ & - & $r q / 4 \wedge$ & - & TN/Tq & - & ميانگين & \\
\hline $\mathrm{TV} / \mathrm{qq}$ & $F T / \Delta$ & $1 / \% F$ & grN/V & rr/Qq & WNTS & $r F / q 1$ & $1 F / \pi$ & $\mid r \wedge / M / M-I f$ & \multirow{3}{*}{ همكاران } \\
\hline १ఎ/А५ & Tr/TQ & $|\omega| \cdot \Delta$ & IV/VE & ra & $1 \% / \Delta$ & $M T / \Delta S$ & $\cdot / \& \wedge$ & $I T M / I T / V$ & \\
\hline $8 / / 19$ & - & $N / r$. & - & $r q / 4 \wedge$ & - & TN/TF & - & ميانگين & \\
\hline
\end{tabular}

جدول ه- مقادير معيارهاى كارايى نش ساتكليف (E) و مجذور ميانخين مربعات خطا (RMSE) در روشهاى مختلف تخمين

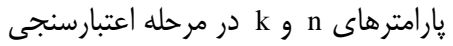

Table 5. The root mean square error (RMSE) and Nash-Sutcliffe (E) indices of each method for estimating $\mathrm{n}$ and $\mathrm{k}$ parameters in the validation phase

\begin{tabular}{|c|c|c|c|c|c|c|c|c|c|c|}
\hline \multicolumn{10}{|c|}{ روشها } & \multirow{3}{*}{ رويداد } \\
\hline \multicolumn{2}{|c|}{ بهونيا وهمكاران } & \multicolumn{2}{|c|}{ ترسيمى هان } & \multicolumn{2}{|c|}{ زمان تا اوج زمان تا عطف } & \multicolumn{2}{|c|}{ تجربى نش } & \multicolumn{2}{|c|}{ ممان } & \\
\hline RMSE & $\mathrm{E}$ & RMSE & $E$ & RMSE & $\mathrm{E}$ & RMSE & $E$ & RMSE & $\mathrm{E}$ & \\
\hline$\Delta / T r$ & $-\cdot / 1$ & $\Delta / r$ &.$- / 11$ & $r / \mu !$ & $\cdot / \Delta)^{c}$ & $\Delta / / \Gamma$ &.$- / \% q$ & $\mathrm{~V} / \cdot 9$ & $-1 / \cdot V$ & 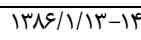 \\
\hline$\cdot / r$. & $-\cdot / \cdot v$ &.$/ T$ & -.1 .9 & $\cdot / T \Lambda$ & $-1 / / Q$ & . & . &.$/ \pi$ & $\cdot / \Delta \mathrm{V}$ & $I T M / I T / V$ \\
\hline$T / V)$ & -.1 .9 & $T / V \Delta$ & -.1 .9 & $1 / \wedge$. &.$- / \mu$ & $K / Q^{\mu}$ & $-\cdot / \cdot+\Delta$ & $r / \varnothing$. & $-\cdot / T \Delta$ & ميانگين \\
\hline
\end{tabular}

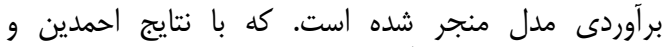

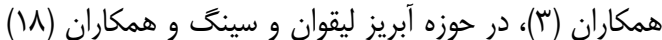

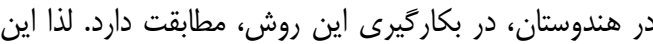

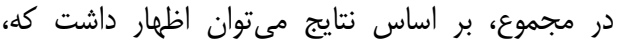

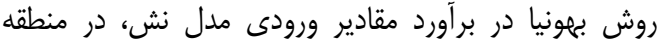

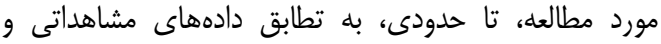




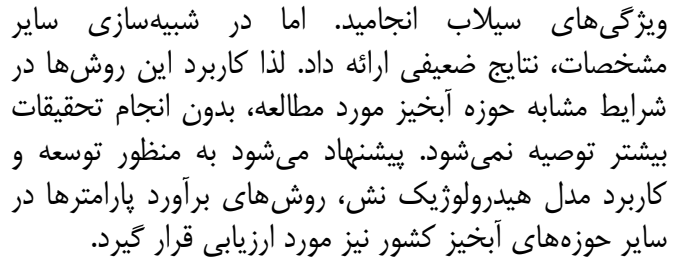

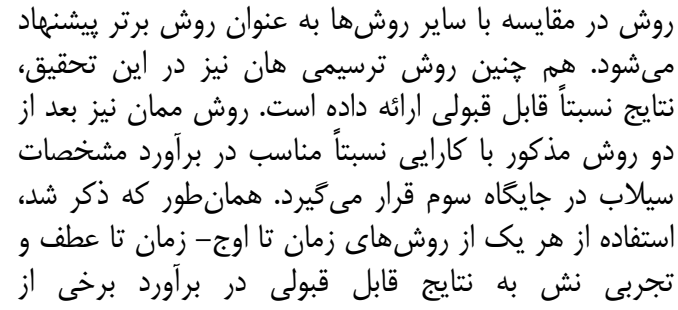

منابع

1. Abdollahian Dehkordi, Z., Kh. Abdollahi, S.J. Sadatinejad, A. Honarbakhsh and M. Nekooeimehr. 2012. The Investigation of direct runoff hydrographs derived from laplace transform and $h 2 u$ instantaneous unit hydrograph models (CaseStudy: Khanmirza Catchment). Journal of Watershed Management Research, 3: 30-42 (In Persian).

2. Agirre, U., M. Guni, J.J. Lopez and F.N. Gimena. 2005. Application of a unit hydrograph based on subwatershed division and comparison with Nash's Instantaneous unit Hydrograph, Catena, 64: 321332.

3. Ahmadin, A., A. Fakheri Fard and A. Gorbani. 2011. Comparsion of hybrid and nash models for derivation of instantaneous unit hydrograph (Case Study: Lighvan Watershed). Journal of Water and Soil Science, 21: 30-41 (In Persian).

4. Bahremand, A.R. and R. Mostafazadeh. 2010. Comparison of different methods for parameter estimation of nash's instantaneous unit hydrograph in jafar-abad watershed. Watershed Management Researches Journal (Pajouhesh \& Sazandegi), 86: 42-51 (In Persian).

5. Bhunya, P.K., S.K. Mishra and R. Berndtsson. 2003. Simplified two-parameter gamma distribution for derivation of synthetic unit hydrograph. Journal of Hydrologic Engineering, 8: 226-230.

6. Chow, V.T., D.R. Maidment and L.W. Mays. 1988. Applied hydrology McGraw-hill, New York.

7. Dong, S.H. 2008. Genetic algorithm based parameter estimation of nash model. Journal of Water Resources Management, 22: 525-533.

8. Fazel modarres, N., F. Niazi, H. Mofid and A. Fakherifard. 2013. Comparison of reservoir-based guhr, nash and hybrid models in derivation of unit hydrograph (Case Study: Kamanaj Olia Watershed). Journal of Water and Soil Science, 22: 103-115 (In Persian).

9. Ghahraman, B. and M. Lotfi. 2010. The applicability of unit hydrograph models in estimating flood hydrograph. Final report of applied research plan (Northern Khorasan Regional Water Company), 117 pp (In Persian).

10. Haan, C.T., B.J. Barfield and J.C. Hayes. 1994. Design hydrology and sedimentology for small catchments. Academic press, San Diego, CA. 588 pp.

11. Mirbagheri, A. 1998. Applied Hydrology, Vol. 1,1 $1^{\text {st }}$ Ed, University of Shiraz Press, Iran , 562 pp. (In Persian)

12. Mostafazadeh, R. and A. Bahremand. 2009. Simulation of direct run-off hydrograph using nash's linear reservoir model in jafar-abad watershed, golestan province. Iran-Watershed Management Science \& Engineering, 3: 9-16 (In Persian).

13. Nourani, V. 2008. A Comparative study on calibration methods of nash's rainfall-runoff model to ammameh watershed, Iran. Journal of Urban and Environmental Engineering, 2: 14-20.

14. Sadeghi, S.H.R. and M. Dehghani. 2006. Efficacy of Estimation Methods for Storage Coefficient of Instantaneous unit Hydrograph in Flood unit hydrograph regeneration. Journal of Agricultural Sciences \& Natural Resources, 13: 152-160 (In Persian).

15. Sadeghi, S.H.R., A. Afzali, M. Vafakhah and A.R. Telvari. 2012. Capability of various statistical analyses in estimation of unit hydrograph components for northern watersheds of Iran. Journal of Watershed Management Research, 3:1-15 (In Persian).

16. Safavi, H.R. 2006. Applied Hydrology. $1^{\text {st }}$ Ed, Arkan Press, Iran, 603 pp (In Persian).

17. Singh, V.P. 1988. Hydrologic Systems. Rainfall-Runoff Modeling, vol. I. Prentice-Hall, Englewood Cliffs, $360 \mathrm{pp}$.

18. Singh, P.K., P.K. Bhunya, S.K. Mishra and U.C. Chaube. 2007. An Extended Hybrid Model for Synthetic unit Hydrograph Derivation. Journal of Hydrology, 336: 347-360.

19. Zakizadeh, F. 2013. Investigating the efficiency of geomorphologic instantaneous unit hydrograph for estimating flood hydrograph characteristics (Case study: Manshad Watershed). M.Sc. Thesis, Yazd University, 119 pp (In Persian). 


\title{
Investigation of the Efficiency of Different Methods for Parameters Estimation of Nash's instantaneous unit Hydrograph in Simulating Flood Hydrograph (Case Study: Manshad Watershed)
}

\author{
Fariba Zakizadeh $^{1}$ and Ali Talebi ${ }^{2}$ \\ 1- Graduated M.Sc., Yazd University (Corresponding author: fariba_zakizadeh@yahoo.com) \\ 2- Associate Professor, Yazd University \\ Received: February 17, $2014 \quad$ Accepted: September 24, 2014
}

\begin{abstract}
In Nash's cascade model that is simple and efficient model for simulating hydrological response in watersheds, catchment behavior is considered same as $\mathrm{n}$ linear reservoirs with $\mathrm{k}$ storage coefficient. In this context, the accuracy of different methods of estimating the Nash model parameters ( $\mathrm{n}$ and $\mathrm{k}$ ) including moment, experimental Nash formula, Han's graphical, time to peak-time to inflection point and Bhunya et al methods have been investigated in Manshad watershed in Yazd province. The $\mathrm{n}$ and $\mathrm{k}$ parameters were calculated using three storm events and physiographic parameters were used for experimental Nash formula. The mean value of calibrated parameters, were used for simulation of second subset of storm events as model validation. The results show that using Bhunya and Han's graphical methods for $\mathrm{n}$ and $\mathrm{k}$ parameters estimation, lead to better agreement between observed and simulated hydrographs. Percentage of relative error in simulating peak discharge, time to peak and flood volume, in Bhunya method, were estimated equal to 28.24, 29.48 \& 8.20\% and in Han's graphical method were estimated equal to $28.29,29.48$ and $8.52 \%$, respectively. Also, the results indicate that time to peak-time to inflection point method and experimental Nash formula give weak results for simulating some flood characteristics. Thus, using these methods in similar catchments is not advised without more researches.
\end{abstract}

Keywords: Bhunya method, Instantaneous unit hydrograph, Manshad watershed, Nash model, Rainfall-runoff modeling, 